\title{
Chemo-dynamical evolution of dwarf galaxies: from flat to cuspy dark matter density profiles
}

\author{
S. Pasetto ${ }^{1,2}$, E. K. Grebel ${ }^{1}$, P. Berczik ${ }^{1}$ and R. Spurzem ${ }^{1}$ \\ ${ }^{1}$ Astronomisches Rechen-Institut, Zentrum für Astronomie der Universität Heidelberg, \\ Germany; ${ }^{2}$ Max-Planck-Institut für Astronomie, Heidelberg, Germany; \\ email: spasetto@ari.uni-heidelberg.de
}

A model of an isolated dwarf spherical galaxy (dSph) is considered in its chemodynamical evolution with time. The system is composed by $3 \gamma$-model density profiles: gas, stellar and dark matter, and it is realized in a spherical symmetric equilibrium configuration. The total masses used in our simulations are covering the dwarf galaxies mass range. The stability of this configuration is first tested for the system evolving under the gravity effect alone and then evolved taking into account for the most relevant stellar astrophysical processes implemented with a Smooth Particle Hydrodynamic approach. The two different kinds of evolution are compared. The dark matter evolves naturally from a centrally cuspy density profile into a flatter one within a timescale of several Gyr. The effect manifests itself naturally, without any tuned initial conditions, as soon as few standard criteria on star formation are assumedand the SN feedback on the ISM has been adopted the prescription in (Cioffi \& Shull 1991) and (Bradamante et al. 1998). This result is expected to be a possible natural explanation for the discrepancy between observations that want flatter dark matter profiles (e.g. de Block 2005), and N-body simulations that predict cuspy dark matter profiles (Navarro et al. 1997). Chemical considerations are presented as a tool to follow with observational parameters the theory predictions.

\section{References}

Pasetto, E. K. Grebel, P. Berczik, \& R. Spurzem, 2009 submitted A\&A and references therein 